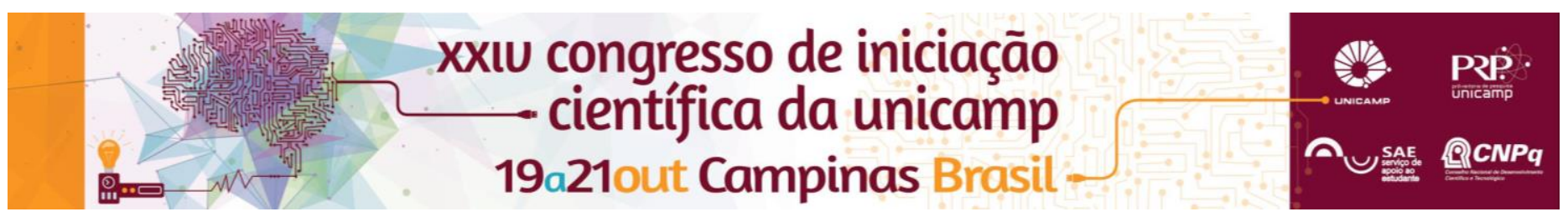

\title{
Laser-capture microdissection for isolation of hippocampal sub-regions and layers for proteomic analysis in an epilepsy animal model
}

\section{Beatriz Bertelli Aoyama (IC), Amanda Morato do Canto (PG), Alexandre Hilario Berenguer Matos (PG), Andre Vieira (PQ), Iscia Lopes Cendes (PQ)}

\begin{abstract}
Mesial temporal lobe epilepsy (MTLE) is the most frequent type of epilepsy in adults and it is usually refractory to clinical treatment. In most patients with MTLE a characteristic histopathological lesion is observed, including hippocampal sclerosis (HS). In this context, the affected hippocampus shows several abnormalities, including neuronal loss. In the present work we aim to use laser-capture microdissection (LCM) to isolate hippocampal sub-regions and layersfrom epileptic rats induced by the classic pilocarpine model. With this material we will perform proteomics analysis to identify abnormal biochemical pathways that could be involved in the etiology of HS in the context of MTLE.
\end{abstract}

Key words: Epilepsy, Hippocampus, Proteomics

\section{Introduction}

Epilepsy is a neurological disorder characterized predominantly by spontaneous and recurrent seizures [1]. In the histopathological aspect the most frequent feature is hippocampal sclerosis (HS), which basically is characterized by the loss of pyramidal neurons in CA1 and CA3 regions [2]. The hippocampus is a structure localized in the mesial temporal lobe, present in the mammalian brain, with functions related to emotional control and memory. It also subdivided in ventral and dorsal regions in rodents and it is composed mainly by two layers, granular (cell bodies) and molecular (dendrites) [3]. Sample preparation for molecular studies from solid tissues such as the brain, represent a real challenge since these tissues are composed by complex structures stemmed from cell types with totally different morphology and function. In this study we aim to isolate hippocampal sub-regions and layers from an epilepsy animal model induced by pilocarpine injections. Isolation of specific regions and layers will be achieved by LCM and used to perform proteomic analysis.

\section{Results and Discussion}

We have induced 5 animals with the pilocarpine protocol $(320 \mathrm{mg} / \mathrm{kg}, \mathrm{IP})$ and 5 controls (Sham). With the frozen tissue we have prepared $60 \mathrm{uM}$-serial sections covering the whole hippocampus, resulting in 20 glass slides (PEN membrane - Life Technologies) with a total of 80 sections. Tissue was laser microdissected for separation of the CA1, CA2, CA3, dentate gyrus and subiculum isolation. In addition, we have isolated the granular and the molecular layer. Tissue was mechanically collected using a surgical microscope and forceps. Subsequently, we have extracted proteins using Urea $8 \mathrm{M}$ and the samples were sent to MS/MS analysis in a LTQ-Orbitrap (CeTICS - Butanta).

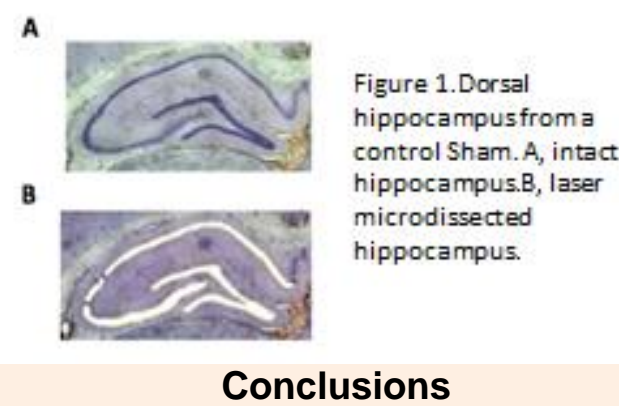

Considering the complexity of brain samples, we can conclude that the proper isolation of the different types of cell population is extremely important. Moreover, the combination of LCM with proteomic tools can allow us to identify new proteins and biological pathways, leading to a better understanding of the molecular mechanisms involved in the epileptogenesis

\section{Acknowledgement}

This work was supported by SponsoredbyCepid-BRAINN/ FAPESP and National Counsel of Technological and Scientific Development (CNPp).

1. Fisher, R. S. et al. Epileptic seizures and epilepsy: definitions proposed by the international League Against Epilepsy (ILAE) and the International Bureau for Epilepsy (IBE). Epilepsia, 2005.

2.Blumcke, I. et al., International consensus classification of hippocampal sclerosis in temporal lobe epilepsy: a Task Force report from the ILAE Commission on Diagnostic Methods. Epilepsia, 2013.

3. Buzsáki, G. \& Moser, E. I. Memory, navigation and theta rhythm in the hippocampal-entorhinal system. NatureNeurosci, 2013. 